\title{
Dynamical imaging of local photovoltage at semiconductor surface by photo-assisted ultrafast scanning electron microscopy
}

\author{
Mohamed Zaghloul ${ }^{1,3 *}$, Silvia M. Pietralunga ${ }^{2,3}$, Gabriele Irde $^{1,3}$, Vittorio Sala ${ }^{1,3}$, Giulio Cerullo ${ }^{1}$, Hao Chen ${ }^{1,3}$, Giovanni \\ Isella $^{4}$, Guglielmo Lanzani ${ }^{1,3}$, Maurizio Zani ${ }^{1}$, and Alberto Tagliaferri ${ }^{1,3}$ \\ ${ }^{1}$ Dipartimento di Fisica, Politecnico di Milano, Piazza Leonardo da Vinci, 32, 20133 Milano, Italy \\ ${ }_{2}^{2}$ Institute for Photonics and Nanotechnologies (IFN) - National Research Council (CNR), Piazza L. da Vinci, 32, 20133 Milano, Italy \\ ${ }^{3}$ CNST@PoliMi, Istituto Italiano di Tecnologia (IIT), Via Giovanni Pascoli 70/3, Milano, Italy \\ ${ }^{4}$ LNESS-Dipartimento di Fisica, Politecnico di Milano, Piazza Leonardo da Vinci 32, 20133 Milano, Italy
}

\begin{abstract}
Photo-assisted Ultrafast Scanning Electron Microscopy (USEM) maps the dynamics of surface photovoltages and local electric fields in semiconducting samples. Photovoltages and their gradients close to surface affect the emission yield and the detection efficiency of secondary electrons (SE), leading to photoexcited SE 2D patterns. In this work, we present a method to characterize the evolution of the patterns up to ultrafast regime. These results reveal the role of surface states in affecting the external field dynamics at picoseconds. Moreover, we show that tiny changes in surface preparation express deeply different photoexcited voltage signals. We investigate the relation between the surface chemistry of Si and photo-induced SE contrast.
\end{abstract}

\section{Introduction}

The study of dynamical Surface Photovoltages (SPV) provides information on the photophysics and charge transport in semiconductors. Recently, techniques of photo-assisted Time-Resolved Scanning Electron Microscopy (TR-SEM) were introduced to provide dynamical 2D mapping of SPV [1,2,3,4]. TR-SEM interpretation is based on the assumption that photoexcited spatial modulations in work function induce secondary electron (SE) contrast patterns. These contrast modulations originate from the photoexcitation of local excess charge carrier distributions, which affect the configuration of surface states and the yield of SE emission. A less considered effect is that this change in charge distribution modifies the electric field outside the surface too, which in turn dynamically changes the SE collection in the SEM $[1,2,4,5,6]$. Both mechanisms, the changes in emission yield and in collection efficiency, contribute to the 2D dynamical SE patterns. Mapping of SPV by TR-SEM provides nanoscopic depth selectivity and lateral resolution, with a broad range of possible view fields and timescales.

Various TR-SEM setups were devised, from the quasisteady-state temporal regime up to ultrafast regime. The latter is usually reached in laser-driven configurations (ultrafast scanning electron microscopy, USEM) based on pulsed electron beams and operated in pump-probe mode $[4,7,8,9,10]$.

TR-SEM has certainly provided interesting insights into the photophysics of semiconductors. Nevertheless, published TR-SEM characterizations on nominally identical surfaces may still lead to controversial and even opposite results.

In the present work, we devise a method for analyzing photo-induced electric fields at semiconductor by 2D TRSEM contrast pattern. Moreover, by operating USEM in ultra-high vacuum (UHV) conditions we performed in-situ chemical analysis of the surface by Auger microspectroscopy and correlate the dynamics of SPVs to the conditions of sample preparation. Our results suggest that, even in the case of nominally identical samples, tiny changes in preparation and in SEM environment in dramatically different TR-SEM dynamics.

\section{Experimental}

Our USEM apparatus is based on a modified UHV $\left(10^{-9} \div 10^{-10}\right.$ torr) SEM (PHI 660), equipped with a $\mathrm{ZrO}$ coated W Field-Effect electron tip. It is driven by a pulsed fiber laser $(300 \mathrm{fs}$ pulses @ $10 \mathrm{MHz}$ repetition rate, $\lambda=1030 \mathrm{~nm})$. The third harmonic (TH) beam $(\lambda=343 \mathrm{~nm})$ is exploited to dynamically excite optical phenomena at semiconductor surfaces. The UHV-SEM is also equipped with an energy analyzer to perform Scanning Auger Microspectroscopy (SAM).

Sample tilting and lateral positioning of the electron detector introduce a symmetry breaking in the detection process that, combined with a proper numerical modeling of the SE collection process, enables us to single out the information on photo-excited electric fields. 
We present here USEM analysis on highly $p$-doped (about $10^{19}$ acceptors $/ \mathrm{cm}^{3}$ ) $\mathrm{Si}(001)$ surfaces, prepared in air by initially sonicating the samples in acetone and ethanol for 20" and then differently treating them in hydrofluoridric acid (HF), to remove native oxide with increasing effectiveness. After this preparation, samples are introduced into the USEM's UHV chamber.

In situ surface chemical composition at $\mathrm{Si}(001)$ is investigated by SAM in UHV at different chemical etching times. Auger spectra have been fitted by linear combinations of spectra taken on differently oxidized $\mathrm{Si}$ surfaces, to correlate USEM contrast with the surface oxygen concentration and get insight into the apparently contrasting results in the USEM literature, where opposite contrast was reported from the surfaces of silicon with similar doping $[7,8]$.

\section{Results and discussion}

\subsection{USEM dynamic imaging}

Our USEM setup operates in pump-probe mode. The TH pulses from the laser source ( sample surface over an elliptical spot (white dashed line in panels (a)-(c) of Fig.1, main axis of about $100 \mu \mathrm{m}$ ). Then an electron pulse (probe) shines the surface at a delayed time (positive when following the photon pulse) and probes the photo-excited effects. Panels (a)-(c) shows typical USEM 2D images, respectively detected at a large negative $(-1 \mathrm{~ns})$, at slightly positive (100 ps) and at large positive $(1.3 \mathrm{~ns})$ delay. The central panel (b), taken at delay close to zero, shows the largest SE contrast change at laser spot, and also in the external area around it.

In order to quantify photo-excited USEM contrast and its dynamics, a differential processing of the USEM images is operated as described hereafter. A multiple-pixel spatial averaging of the measured SE intensity is performed over a defined range, named hereafter area of interest (AOI). The AOI is a sub-portion of the width of the area where photo-excited effects are evident. A reference contrast value is also obtained by averaging SE intensity measured on another area, identical in shape to AOI and located in an unperturbed region far away from the laser spot. The USEM contrast is finally obtained as the difference between the AOI average and the reference contrast. By scanning the AOI over the image frame, 2D USEM contrast maps are obtained. Panel (e) of Fig. 1 shows a map acquired around zero delay on a non-HF etched sample surface (shown in Fig. 1.b). It is clear from Fig. 1.e that USEM contrast behaves differently on points at the left side (left area) and at the right side (right area) of the laser spot. Two regions of maximal contrast exist, positive on the left hand side of the laser spot (at the center of the image) and negative at the right hand side. The results for the red AOI at left and for the black AOI at right are plotted as a function of pump-probe delay in panel (d) of Fig. 1.

All of the temporal plots show a step-like evolution of the USEM contrast within 100 ps from zero delay, followed by a slower decay on a few ns timescale.

The state-of-the-art USEM literature focuses on the SE dynamics measured at the center of the laser spot and
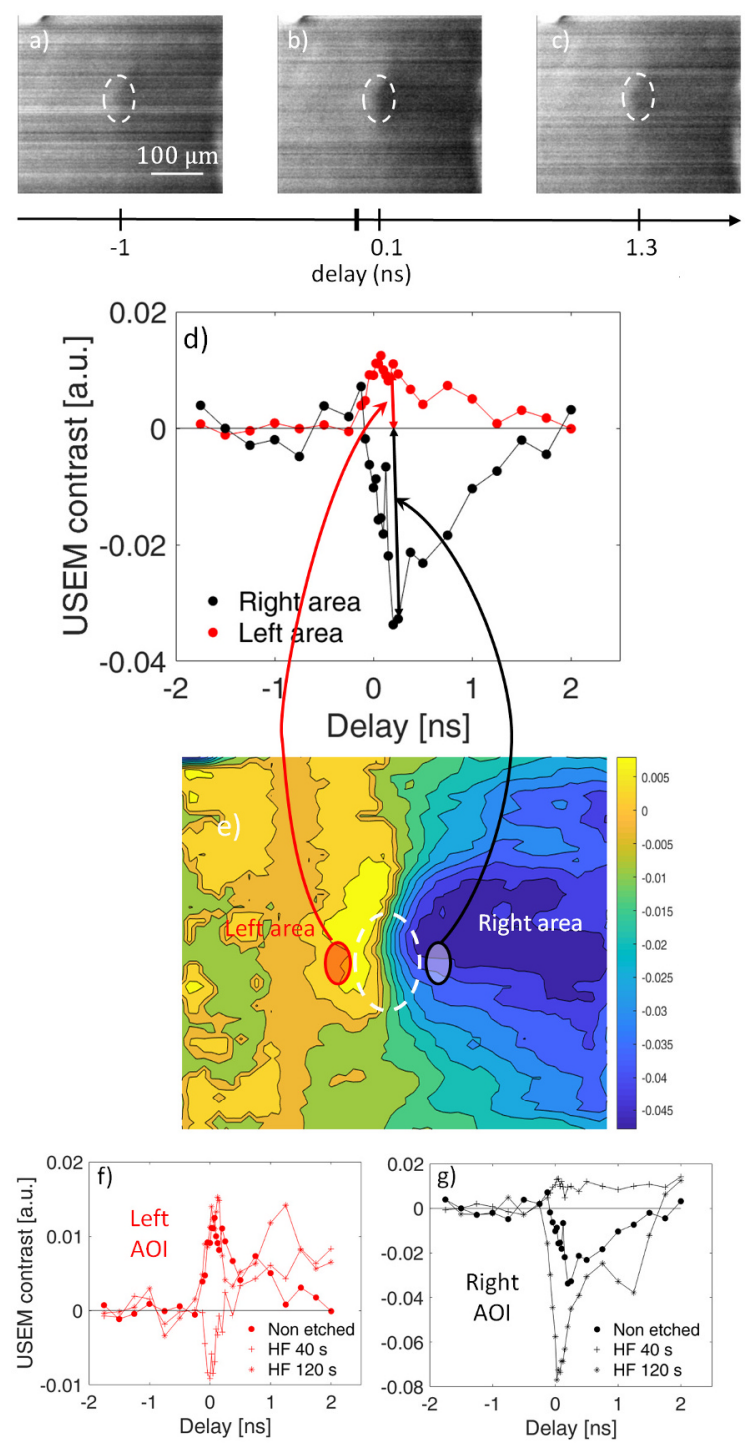

Fig. 1. Time evolution of USEM photoinduced contrast on highly $p$-doped $\mathrm{Si}$, as a function of pump-probe delay. (a)-(c): 2D USEM contrast maps on a non-HF etched sample at three delay times, highlighting the bipolarity in sign of the contrast. (d) Time series of the USEM contrast, from the two AOI at left (red) and right (black) side of the laser spot (white), shown on the 2D USEM contrast step map in panel (e). (f): comparison of the SE contrast amplitude induced in differently HF treated samples in the left AOI; $(\mathrm{g})$ the same for the right AOI.

relates it to the SE emission yield $[3,7,8,10]$. In our setup, a spatial bipolar modulation of the SE signal around the laser spot appears, which we attribute to the breaking of the cylindrical symmetry with respect to surface normal axis by the side SE detector. This modulation appears in the 2D patterns in Fig. 1, panels (a)-(c) (SE images) and panel (e) (USEM contrast map). We show that on highly $p$-doped $\mathrm{Si}$ surfaces, the USEM time series may have very different maximum amplitudes, and even switch in sign depending on SE emission point. This switching in sign cannot be explained by an spatial intensity modulation of $\mathrm{SE}$ emission yield within the laser spot itself. On the contrary, 
it mostly derives from the interplay between the gradients of the SPV and symmetry-breaking in detection. A positive SPV in the laser spot corresponds to a right-to-left electric field component parallel to the sample surface at the left side of the laser spot. As a consequence, the trajectories of SE emitted on the left side bend towards the detector positioned at the right, and their collection enhances. An opposite behavior appears at the right side, where the SE trajectories bend away and the collection reduces $[1,2]$. This switching in sign of the USEM contrast at the sides of the laser spot is therefore a fingerprint of the external fields at sample surface and has the potential to provide qualitative (sign) and quantitative (intensity) insight on SPVs. A similar effect was recently reported on GaAs surfaces [4].

The impact of the surface preparation on USEM contrast and on SPV is evident from Fig. 1, panels (f) and $(\mathrm{g})$, where the result on three differently prepared highly $p$-doped $\mathrm{Si}(001)$ surfaces is compared. The first sample was just sonicated in acetone and ethanol, without HF etching (leaving the native oxide on the surface untouched); the second and third ones were subsequently etched in HF for $40 \mathrm{~s}$ and $120 \mathrm{~s}$, respectively. On each surface, the inversion of the contrast amplitude with the displacement of AOI from the left hand (red) to the right hand side (black) of the laser spot is confirmed. As a very remarkable feature, we notice that the time series from the three surfaces show dramatic differences in amplitude and shape, with the 40" HF etched one presenting even a change in sign of the step with respect to the other two. This data reveals the sensitivity of the time varying photovoltages to the surface structure and to surface electronic states.

\subsection{Auger electron spectroscopy analysis}

Scanning Auger Microspectroscopy has been performed on samples after doing USEM measurements to analyze chemical differences in the highly $p$-doped $\mathrm{Si}(001)$ surfaces mentioned above and to relate them to the different dynamics shown by USEM signal as a function of surface preparation. Auger KLL lines for carbon, oxygen and silicon are reported in Fig. 2. The positive-to-negative peak intensity of those differential spectra is a monotonic function of the surface atomic concentration of the elements. Panel (a) shows carbon KLL line, featuring the negative peak around $269 \mathrm{eV}$. The C KLL intensity from the non-etched surface is a small fraction of the one from the two etched ones. Moreover, it is remarkably more intense from the surface HF etched for 120", than in the 40" HF etched one. Panel (b) shows the oxygen KLL negative peak around $510 \mathrm{eV}$. The O KLL more intense for non-etched with respect to the two etched surfaces, where it features a nearly equal height. The spectra of the Si KLL at about $1630 \mathrm{eV}$ in panel (c) show almost the same strength in the non-etched and in the 40" HF etched surface, while its intensity is remarkably lower in the 120" $\mathrm{HF}$ etched one. In Fig. 2 it is noteworthy that the O KLL is less intense, while C KLL appears more intense after the etching process for both 40 " and 120' This is an evidence of the removal of the native $\mathrm{SiO} 2$ layer by the HF etching, and at the same time of the increase in carbon
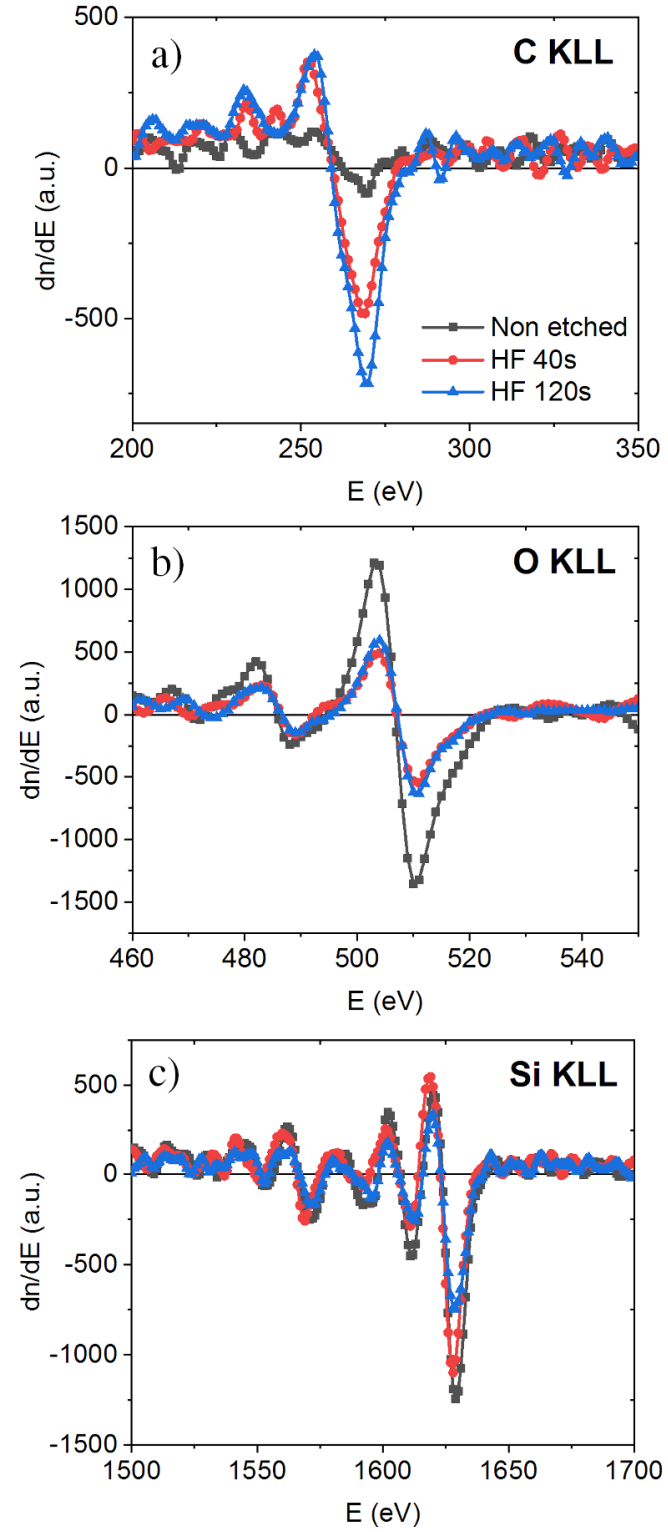

Fig. 2. Auger KLL spectra for highly $p$-doped Si surfaces (nonetched, 40" and 120" HF etching): (a) carbon, (b) oxygen and (c) silicon.

contamination of the surface during the post-etching time period until the sample is inserted in the vacuum chamber. This carbon contamination is more effective on the 120" $\mathrm{HF}$ etched than on the 40" HF etched surface. This is supported by the lower intensity of the Si KLL line in the former, which is tentatively attributed to the lower $\mathrm{Si}$ concentration in the carbon-rich over-layer at the surface. We report that carbon surface concentration is not influenced by the USEM measurements, as the Auger spectra do not show measurable changes on the e-beam exposed surface with respect to the as-inserted surface (not shown). 


\subsection{Correlation between USEM and SAM}

The correlation between the USEM contrast reported in Fig 1.f and the atomic concentrations of carbon and oxygen at surface from the Auger spectra in Fig. 2 highlights the sensitivity of the USEM technique to SPVs, and in turn to the surface electronic structure as resulting from its chemical and structural state. USEM contrast 2D pattern in Fig 1.f reveals the same sign when measured on the non-etched surface and on the 120" HF etched one, while an opposite sign is evident on the 40 " $\mathrm{HF}$ etched. Our interpretation of the influence of USEM contrast with duration time of chemical etching, is that the etching itself and the following exposure to air determine very different chemical and structural states of the $\mathrm{Si}(001)$ surface. This in turn deeply affects the value of the work function and of the charge carrier transport at surface that result in different photovoltages and even in their sign reversal.

\section{Conclusion}

The dynamics of photo-induced voltages at the (001) surface of highly $p$-doped silicon has been investigated by ultrafast scanning electron microscopy (USEM) and Scanning Auger Microspectroscopy (AES). A protocol to analyze differential USEM contrast maps was developed, to quantify the photo-induced contrast. USEM contrast is strongly influenced by chemical state resulting from the preparation of the $\mathrm{Si}(001)$ surface. Different HF etching times lead even to a switching in sign of the USEM contrast pattern. By correlating USEM and Auger electron microspectroscopy on differently prepared samples, we highlight the dramatic role of surface chemistry on photovoltages and charge dynamics at $\mathrm{Si}(001)$ surface.

\section{References}

1. G. Irde, S. M. Pietralunga, V. Sala, M. Zani, J.M. Ball, A.J. Barker, A. Petrozza, G. Lanzani, A. Tagliaferri, Micron 121, 53 (2019)

2. S.M. Pietralunga, G. Irde, A.J.A.J. Barker, J.M. Ball, A. Petrozza, V. Sala, M. Zani, G. Lanzani, A. Tagliaferri, Adv. Mater. Interfaces 7(16), 2000297 (2020)

3. Y. Li, U. Choudhry, J. Ranasinghe, A. Ackerman, B. Liao, J. Phys. Chem. A 124(25), 5246-52 (2020)

4. M.W.H. Garming, M. Bolhuis, S. Conesa-Boj, P. Kruit, J.P. Hoogenboom, J. Phys. Chem. Lett. 11(20), 8880-6 (2020)

5. W. Han, M. Zheng, A. Banerjee, Y.Z. Luo, L. Shen, A. Khursheed, Sci. Rep. 10(1), 22144 (2020)

6. A. Khursheed, Secondary Electron Energy Spectroscopy in the Scanning Electron Microscope, (World Scientific, 2020)

7. E. Najafi, T. Scarborough, J. Tang, A.H. Zewail, Science 347(6218), 164 (2015)

8. E. Najafi, V. Ivanov, A. Zewail, M. Bernardi, Nat. Commun. 8(1), 15177 (2017)
9. M. Zani, V. Sala, G. Irde, S. M. Pietralunga, C. Manzoni, G. Cerullo, G. Lanzani, and A. Tagliaferri, Ultramicroscopy, 187, 93 (2018)

10. E. Najafi, A. Jafari, Appl. Phys. J. 125, 185303 (2019) 